\title{
Combination of theoretical and in situ experimental investigations of the role of lithium dopant in manganese nitride: a two-stage reagent for ammonia synthesis
}

\author{
Said Laassiri, (D)*abc Constantinos D. Zeinalipour-Yazdi, (DD d \\ Nicolas Bion, ${ }^{e}$ C. Richard A. Catlow ${ }^{f g}$ and Justin S. J. Hargreaves ${ }^{b}$
}

Received 8th December 2019, Accepted 22nd January 2020

DOI: $10.1039 /$ c9fd00131j

Manganese nitride related materials are of interest as two-stage reagents for ammonia synthesis via nitrogen chemical looping. However, unless they are doped with a cocation, manganese nitrides are thermochemically stable and a high temperature is required to produce ammonia under reducing conditions, thereby hindering their use as nitrogen transfer materials. Nevertheless, when lithium is used as dopant, ammonia generation can be observed at a reaction temperature as low as $300{ }^{\circ} \mathrm{C}$. In order to develop strategies for the improvement of the reactivity of nitride materials in the context of two-stage reagents, it is necessary to understand the intrinsic role of the dopant in the mechanism of ammonia synthesis. To this end, we have investigated the role of lithium in increasing the manganese nitride reactivity by in situ neutron diffraction studies and $\mathrm{N}_{2}$ and $\mathrm{H}_{2}$ isotopic exchange reactions supplemented by DFT calculations.

\section{Introduction}

In the design of a novel generation of materials for ammonia synthesis, metal nitrides have significant potential for the development of very active catalysts that could operate under less severe conditions than the conventional Haber Bosch Process. While the mechanism of ammonia synthesis is still not unequivocally

${ }^{a}$ Univ. Lille, CNRS, ENSCL, Centrale Lille, Univ. Artois, UMR 8181, UCCS - Unité de Catalyse et Chimie du Solide, F-59000, Lille, France.E-mail: Said.laassiri@um6p.ma

${ }^{b}$ WestCHEM, School of Chemistry, University of Glasgow, Glasgow, G12 8QQ, UK

${ }^{c}$ Chemical \& Biochemical Sciences, Green Process Engineering (CBS), Mohamed VI Polytechnic University, UM6P, 43150, Ben Guerir, Morocco

${ }^{d}$ School of Science, University of Greenwich, Central Avenue, Chatham Maritime, Kent, ME4 4TB, UK ${ }^{e}$ Institut de Chimie des Milieux et Matériaux de Poitiers (IC2MP), University of Poitiers, CNRS, 4 Rue Michel Brunet, TSA51106, F86073 Poitiers Cedex 9, France

${ }^{f}$ School of Chemistry, Cardiff University, Park Place, Cardiff CF10 1AT, UK

${ }^{g}$ Department of Chemistry, University College London, 20 Gordon Street, London, WC1H OAJ, UK 
elucidated for nitride based materials, lattice nitrogen mobility and reactivity have been demonstrated in a number of nitride materials including mono- and bimetallic nitrides such as $\mathrm{Ta}_{3} \mathrm{~N}_{5}, \mathrm{Mg}_{3} \mathrm{~N}_{2}$ and $\mathrm{Co}_{3} \mathrm{Mo}_{3} \mathrm{~N}$, leading to the possibility of ammonia production through pathways based upon the Mars-van Krevelen (MvK) mechanism. ${ }^{1-4}$ In this mechanism, some of the lattice nitrogen can react directly with dihydrogen to produce ammonia, resulting in the generation of nitrogen vacancies that can activate $\mathrm{N}_{2}$ to complete the cycle.

Over the years, oxide materials operating via the MvK mechanism have inspired the development of chemical looping technology for combustion reactions. The idea is based upon separating the reaction into different steps where the combustion stage occurs in the absence of dioxygen and the oxygen required for the reaction is provided by an oxide-based material. The oxygen-depleted material is subsequently regenerated in a second reaction step. The possibility to deconstruct processes into multiple sub-reactions conducted under different conditions (such as temperature, pressure and gas composition) is an advantage of looping material design when compared to catalytic material design. Considering the thermodynamic limitation of the ammonia synthesis reaction, the application of the chemical looping principle in ammonia synthesis presents an interesting alternative to the Haber Bosch Process. In a similar manner to the oxide based chemical looping processes, ammonia synthesis can be deconstructed into sub-reactions undertaken in the presence of a nitride material acting as a nitrogen transfer agent, where the nitride can be reduced in a first step to produce ammonia and its resultant nitrogen deficiency replenished in a second step using $\mathrm{N}_{2} \cdot{ }^{5,6}$ The sub-reactions could be conducted under different conditions which could alleviate some of the limitations related to the adsorption and dissociation of dinitrogen in the catalytic cycle. Amongst materials that can act as nitrogen transfer materials, manganese has attracted a degree of attention due to its ability to activate molecular dinitrogen at moderate temperature and ambient pressure to form a range of nitrides with different crystal structure and stoichiometry, including $\mathrm{Mn}_{4} \mathrm{~N}, \mathrm{Mn}_{3} \mathrm{~N}_{2}$ and $\mathrm{Mn}_{6} \mathrm{~N}_{5+x} \cdot{ }^{7,8}$ Furthermore, previous studies have shown that the lattice nitrogen in manganese nitride is both labile and reactive under reducing conditions. ${ }^{5,9}$ When manganese nitride is reacted with hydrogen at ambient pressure, ammonia is produced. The possibility of manganese based systems to "breathe in" nitrogen and "breathe out" ammonia by simply cycling between different reaction environments at ambient pressure offers an interesting route for the development of alternative approaches for ammonia generation under milder reaction conditions. However, the thermochemical stability of manganese nitride and its apparent low selectivity towards ammonia generation hinder its application as a nitrogen transfer material. In order to improve the reactivity of nitride materials, we have demonstrated that doping with small amounts of lithium containing dopant results in high reactivity towards hydrogen at a reaction temperature as low as $300{ }^{\circ} \mathrm{C} .{ }^{5} \mathrm{~A}$ related effect has been reported in the catalytic ammonia synthesis reaction, where the addition of $\mathrm{LiH}$ to transition metal-based materials as co-catalyst facilitated the production of ammonia at $350{ }^{\circ} \mathrm{C}^{\mathbf{1 0}}$

Historically, the development of highly active catalysts for ammonia synthesis is closely related to the use of suitable dopants to enhance performance. For example, in the case of promoted iron catalysts, $\mathrm{Al}_{2} \mathrm{O}_{3}$ is used as a structure promoter. The difference in terms of accessible surface area between promoted 
and bare iron catalysts is twenty-fold. However, the role of dopants is not only restricted to structural modification, but also includes modification of the electronic properties. In the case of the industrial ammonia catalyst, the addition of $\mathrm{K}_{2} \mathrm{O}$ in the doubly promoted iron catalyst considerably increases catalytic activity at higher pressure when compared to the singly promoted catalyst. ${ }^{11}$ In the context of the $\mathrm{Ru}$ based catalyst system, which is very active for ammonia generation at low pressure, density functional theory (DFT) indicated that the addition of $\mathrm{Cs}^{+}$on the $\mathrm{Ru}(0001)$ surface reduced the $\mathrm{N}_{2}$ dissociation barrier and the coverage of other intermediates along the reaction path. ${ }^{11}$ However, to the authors' current knowledge, the role of lithium doping in both catalytic and chemical looping ammonia synthesis is not yet clearly understood.

In order to investigate the origin of the increase in manganese reactivity upon lithium doping, a series of in situ neutron diffraction studies and $\mathrm{N}_{2}$ and $\mathrm{H}_{2}$ isotopic exchange reactions were performed and were supplemented by DFT calculations. Initially, a series of in situ neutron diffraction studies was conducted to provide information on the structural evolution of lithium doped and nondoped manganese nitride materials under reducing reaction conditions. Furthermore, the role of lithium in the process of hydrogen and nitrogen activation was studied by isotopic exchange reactions, while detailed DFT calculations provide insight into the energetic aspects of the interaction between the reactant and the surface in the presence and absence of the dopant.

\section{Methods and materials}

\subsection{Experimental methods}

2.1.1 Material synthesis. The synthesis procedures applied for the preparation of undoped and Li-doped manganese nitrides have been described in detail elsewhere.,12 In summary, $0.3 \mathrm{~g}$ of $\mathrm{Mn}_{2} \mathrm{O}_{3}$ (Sigma Aldrich, 99\%) and $1.0 \mathrm{~g}$ of $\mathrm{NaNH}_{2}$ (Sigma Aldrich, 98\%) were loaded into a Teflon-lined steel autoclave inside a nitrogen-filled glovebox. The autoclave was tightly closed and kept in an oven at $240{ }^{\circ} \mathrm{C}$ for $36 \mathrm{~h}$. The product was then collected by filtration, washed with ethanol and water, and dried overnight at room temperature. For the preparation of Li doped manganese nitride, first an oxide precursor was prepared by a conventional co-precipitation route, using aqueous ammonia as precipitation agent, followed by calcination under static air at $800{ }^{\circ} \mathrm{C}$ for $8 \mathrm{~h}$. The nitride phase was then prepared in a similar manner to the non-doped manganese nitride. The results of structural and textural characterisation have been presented previously. ${ }^{5}$

2.1.2 Isotopic exchange experiments. Isotopic exchange experiments were performed in an apparatus described elsewhere. ${ }^{13}$ In summary, the system comprised a closed-loop reactor coupled to a mass spectrometer for monitoring the composition of the gas evolved. A recirculating pump was connected to the system to minimise gas phase diffusion limitations. Temperature-programmed isotopic exchange and isothermal isotopic exchange experiments were undertaken on $100 \mathrm{mg}$ of the manganese nitride materials. Prior to the isotopic exchange experiments, samples were subjected to an in situ $3: 1 \mathrm{H}_{2}: \mathrm{N}_{2}$ activation step at $700{ }^{\circ} \mathrm{C}$ for $1 \mathrm{~h}$.

2.1.2.1 Nitrogen activation process. The study of the nitrogen activation process was performed using labeled nitrogen ${ }^{15} \mathrm{~N}_{2}(98 \%+$ purity, supplied by 
Cambridge Isotope Laboratories, Inc.). The mass-to-charge ratios of 28, 29 and 30 $\mathrm{m} / \mathrm{z}$ were monitored as a function of time to follow the process.

2.1.2.2 Hydrogen activation process. Hydrogen activation was studied via the $\mathrm{H}_{2} / \mathrm{D}_{2}$ exchange reaction. Isothermal homoexchange experiments were conducted at temperatures ranging between $100{ }^{\circ} \mathrm{C}$ and $300{ }^{\circ} \mathrm{C}$. After the activation step, $\mathrm{D}_{2}$ (20 mbar) and $\mathrm{H}_{2}$ (20 mbar) were introduced in the system and the evolution of the partial pressures of reactants and products was monitored by mass spectrometry.

2.1.3 In situ powder neutron diffraction. In situ controlled atmosphere high temperature time-of-flight (ToF) PND data was collected using the high intensity Polaris diffractometer at the ISIS pulsed spallation source (Rutherford Appleton Laboratory, UK). Powder samples ( $c a .1 \mathrm{~g}$ ) were loaded into $11 \mathrm{~mm}$ diameter thin walled stainless steel cells and held in place between quartz glass frits to permit a flow of reaction gas to circulate through the samples during data collection. The cell assembly was mounted in a dedicated neutron diffraction furnace capable of heating it from room temperature to $\sim 900{ }^{\circ} \mathrm{C}$ and connected to a gas panel fitted with mass flow controllers allowing a mixture of gases to flow through the sample at controlled rates during data collection. A collimator manufactured from neutron-absorbing boron nitride ceramic was placed around the cell and enabled diffraction patterns to be collected in the Polaris $2 \theta \approx 90^{\circ}$ detector bank which were free of Bragg reflections from the steel walls of the cell. Rietveld refinements against the PND data were performed using the General Structure Analysis System (GSAS) through the EXPGUI interface. ${ }^{\mathbf{1 4 , 1 5}}$

\subsection{Density functional theory}

All DFT calculations were periodic $\Gamma$-point ${ }^{16}$ and spin-polarised calculations obtained with the use of the VASP 5.4.1 code. ${ }^{17,18}$ Exchange and correlation effects were considered within the generalized gradient approximation (GGA) using the revised Perdew-Burke-Ernzerhof (revPBE) exchange-correlation (XC) functional, ${ }^{19}$ with the projector augmented-wave (PAW) method $^{20,21}$ used to represent core states. These were $1 \mathrm{~s}$ to $3 \mathrm{p}$ for $\mathrm{Mn}$ and $1 \mathrm{~s}$ for $\mathrm{N}$ and Li. Geometry optimizations were performed with a residual force threshold on each atom of $0.02 \mathrm{eV}^{-1}$, using the conjugate-gradient algorithm. The convergence criterion for electronic relaxation was $10^{-1} \mathrm{eV}$. The initial charge density was obtained by superposition of atomic charges. Various initial adsorption configurations were considered

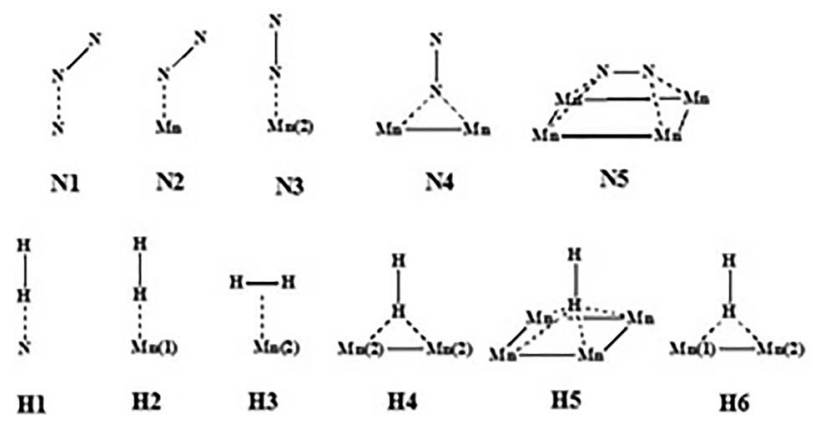

Scheme 1 Potential $\mathrm{N}_{2}$ and $\mathrm{H}_{2}$ adsorption sites on the (100) $\eta-M n_{3} N_{2}$ surface. 
where the adsorbate was either in a side-on or in an end-on configuration, and the adsorbate bond length for $\mathrm{N}_{2}\left(r_{\mathrm{N}-\mathrm{N}}\right)=1.11 \AA$, and that for $\mathrm{H}_{2}\left(r_{\mathrm{H}-\mathrm{H}}\right)=0.75 \AA$. The various adsorption sites were the symmetry unique surface sites shown in Scheme 1 , which resulted in 8 sites. The adsorption energy was taken as the total energy difference between the fully relaxed bound state of the surface-adsorbate complex and the fully relaxed surface slab and the isolated molecules given by eqn (1),

$$
\Delta E_{\mathrm{ads}, \mathrm{D} 3}=E_{\text {slab-X }}-E_{\text {slab }}-E_{\mathrm{X}}
$$

where $\mathrm{X}=\mathrm{N}_{2}, \mathrm{H}_{2}$ and Li. Grimme's dispersion corrections were included via the zero-damping DFT-D3 correction method, ${ }^{22}$ in which the following dispersion energy correction is added to the Kohn-Sham energies,

$$
E_{\text {disp }}=-\frac{1}{2} \sum_{i=1}^{N} \sum_{j=1}^{N}\left(s_{6} \frac{C_{6, i j}}{r_{i j}{ }^{6}}+s_{8} \frac{C_{8, i j}}{r_{i j}{ }^{8}}\right),
$$

where $C_{6, i j}$ and $C_{8, i j}$ denote the averaged (isotropic) $6^{\text {th }}$ and $8^{\text {th }}$ order dispersion coefficients for atom pair $i j$, and $r_{i j}$ is the internuclear distance between atoms $i$ and $j . s_{6}$ and $s_{8}$ are the functional-dependent scaling factors. The D3 correction was invoked in calculating the adsorption energies, as it is generally known that the revPBE is unable to completely recover dispersion interactions as a result of polarisation effects primarily of the transition metals (TMs).

\section{Results and discussion}

\subsection{Structural evolution of manganese nitride materials under reducing conditions: in situ neutron diffraction}

3.1.1 Nitride phase identification: starting material. The neuron diffraction data collected for the manganese nitride materials are presented in Fig. 1 and 2. Rietveld refinement against the PND data was performed using the previously published $\theta-\mathrm{Mn}_{6} \mathrm{~N}_{5+x}$ structure as the starting model. Considering that $\theta-\mathrm{Mn}_{6} \mathrm{~N}_{5+x}$

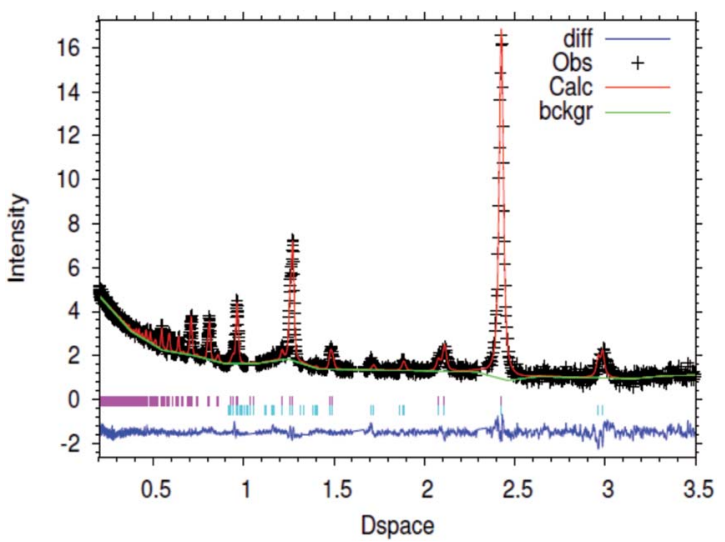

Fig. 1 Fitted powder neutron diffraction profile from Rietveld refinement for the $1 \%$ wt Li/ $\mathrm{Mn}_{6} \mathrm{~N}_{5+x}$ sample at ambient temperature. 


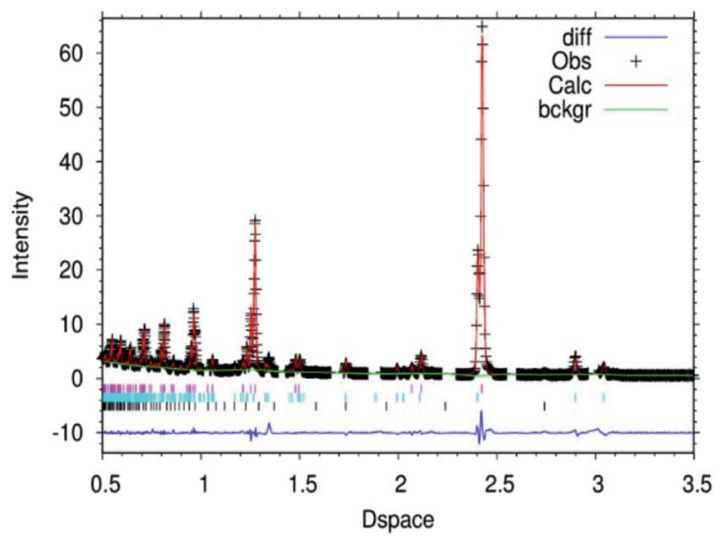

Fig. 2 Fitted powder neutron diffraction profile from Rietveld refinement for the $\theta$ $\mathrm{Mn}_{6} \mathrm{~N}_{5+x}$ sample at ambient temperature.

exhibits an antiferromagnetic ordering of the first kind, the magnetic structure was considered as a second phase using the magnetic symmetry group $F C 2^{\prime} / \mathrm{m}^{8}$

In the case of the lithium doped material, the crystal structure derived from the Rietveld refinement, Fig. 1, corresponded closely to the to the $\theta-\mathrm{Mn}_{6} \mathrm{~N}_{5+x}$ structure as described by Leineweber et al. ${ }^{8}$ In summary, it presented a defective $\mathrm{NaCl}$ type structure with tetragonal distortion $(\mathrm{F} 4 / \mathrm{mmm})$. The overall details of the refinement are summarized in Table 1 . In the case of the non-doped manganese nitride, the material was found to comprise two phases, being predominantly composed of $\theta-\mathrm{Mn}_{6} \mathrm{~N}_{5+x}$ and to a lesser extent of $\eta-\mathrm{Mn}_{3} \mathrm{~N}_{2}\left(\theta-\mathrm{Mn}_{6} \mathrm{~N}_{5+x} / \mathrm{Mn}_{3} \mathrm{~N}_{2}=1\right.$ / 0.2 weight ratio) as extracted from the Rietveld refinement as presented in Fig. 2. In the lithium doped manganese nitride, only the $\theta-\mathrm{Mn}_{6} \mathrm{~N}_{5+x}$ phase was observed, indicating that the addition of lithium favours the formation of $\theta-\mathrm{Mn}_{6} \mathrm{~N}_{5+x}$. The application of powder neutron diffraction provides a much greater insight into the phase complexity of these materials, for which the powder X-ray diffraction pattern was assigned previously to $\mathrm{Mn}_{3} \mathrm{~N}_{2}{ }^{5}$

3.1.2. In situ neutron diffraction studies under reducing conditions. The structural evolution of both the non-doped and lithium doped manganese nitride materials during lattice nitrogen discharge was followed by in situ neutron diffraction under $60 \mathrm{~mL} \mathrm{~min}^{-1} 75 \mathrm{vol}^{\circ} \mathrm{H}_{2}$ in $\mathrm{Ar}$ (BOC, 99.98\%) to reproduce the conditions of lattice nitrogen reduction in chemical looping. All diffraction

Table 1 Summary of crystallographic parameters of the $1 \%$ wt Li/Mn ${ }_{6} \mathrm{~N}_{5+x}$ system derived from room temperature neutron diffraction data

\begin{tabular}{lll}
\hline & Nuclear structure & Magnetic structure \\
\hline Space group & $F 4 / m m m$ & $F C 2^{\prime} / m^{\prime}$ \\
Cell parameter $a / \AA$ & $4.2195(3)$ & $4.2195(3)$ \\
Cell parameter $c / \AA$ & $4.1514(4)$ & $4.1514(4)$ \\
Occupancy for $\mathrm{N}$ site & $1.00 \pm 0.02$ & \\
$R_{\mathrm{p}}$ & 0.041 & \\
$\mathrm{w} R_{\mathrm{p}}$ & 0.056 & \\
$\chi^{2}$ & 1.396 &
\end{tabular}


patterns were analysed by the Rietveld method using $\theta-\mathrm{Mn}_{6} \mathrm{~N}_{5+x}$ and/or $\eta-\mathrm{Mn}_{3} \mathrm{~N}_{2}$ as starting models.

Fig. 3 presents a selection of in situ diffraction patterns collected at $400{ }^{\circ} \mathrm{C}$ for the undoped $\theta-\mathrm{Mn}_{6} \mathrm{~N}_{5+x}$ sample. Following the reaction no structural changes were apparent in both the $\theta-\mathrm{Mn}_{6} \mathrm{~N}_{5+x}$ and the $\eta-\mathrm{Mn}_{3} \mathrm{~N}_{2}$ phase fractions, confirming the thermochemical stability of manganese nitrides during the discharge reaction as had been reported earlier in the literature. ${ }^{5}$ Furthermore, no changes were observed upon increasing the reaction temperature to $500{ }^{\circ} \mathrm{C}$.

Fig. 4 presents in situ neutron diffraction patterns of the lithium doped manganese nitride material collected at different times on stream at $400{ }^{\circ} \mathrm{C}$. At low temperature, $\leq 200{ }^{\circ} \mathrm{C}$, no noticeable changes were detected in the sample and only the $\theta-\mathrm{Mn}_{6} \mathrm{~N}_{5+x}$ phase was apparent. This observation is consistent with the low reactivity of $\theta-\mathrm{Mn}_{6} \mathrm{~N}_{5+x}$ for ammonia production under reducing conditions at reaction temperatures below $300{ }^{\circ} \mathrm{C} .{ }^{5}$ However, upon increasing the temperature, a linear decrease in the nitrogen occupancy was observed, indicating reduction of the $\theta-\mathrm{Mn}_{6} \mathrm{~N}_{5+x}$ proceeding through a nitrogen vacancy creation pathway. However, when the concentration of nitrogen vacancies reached a critical concentration $(n=0.88)$, the reduction of the $\theta-\mathrm{Mn}_{6} \mathrm{~N}_{5+x}$ was accompanied by a structural rearrangement, resulting in two inequivalent $\mathrm{Mn}$ sites, with the resulting structure being related to $\eta-\mathrm{Mn}_{3} \mathrm{~N}_{2}$.

\subsection{Effect of $\mathrm{Li}$ in the $\mathrm{N}_{2}$ and $\mathrm{H}_{2}$ activation processes: isotopic exchange experiment}

3.2.1. $\mathbf{N}_{2}$ activation process. The ability of the manganese nitride related materials to activate molecular nitrogen was studied via heterolytic temperature programmed nitrogen isotopic exchange (TPNIE) in the range of $400{ }^{\circ} \mathrm{C}$ to $700{ }^{\circ} \mathrm{C}$ as presented in Fig. 5. The results of the TPNIE experiment, shown in Fig. 5(a), indicate

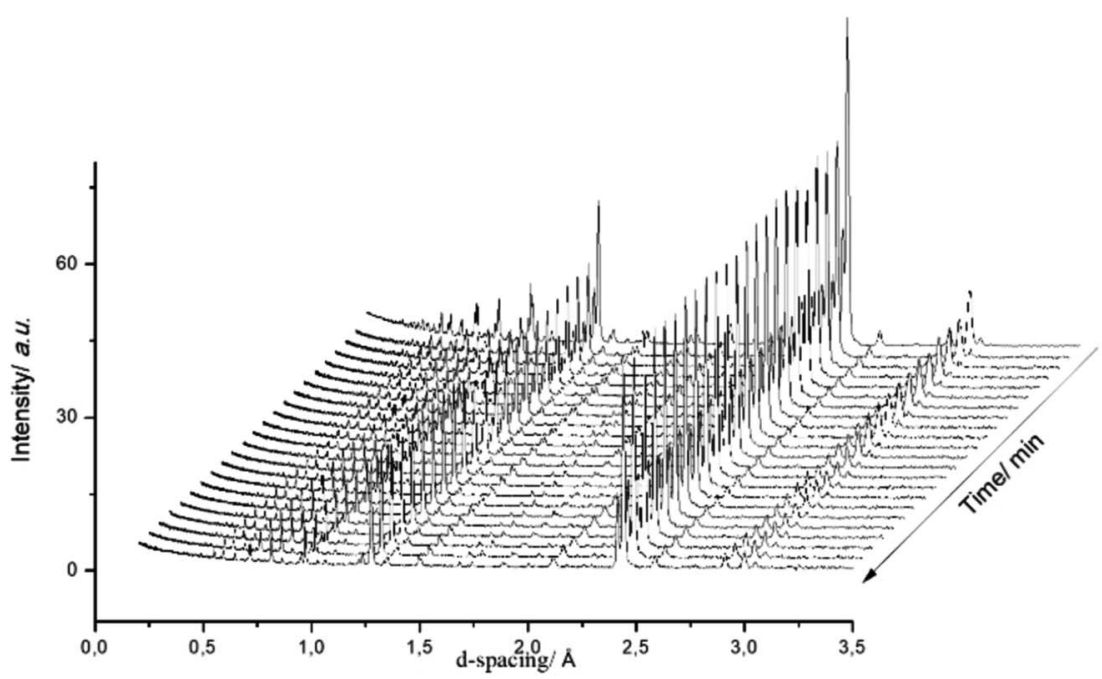

Fig. 3 Diffraction patterns of $\theta-\mathrm{Mn}_{6} \mathrm{~N}_{5+x}$ during the discharge reaction at $400{ }^{\circ} \mathrm{C}$. In situ measurements were conducted under $60 \mathrm{~mL} \mathrm{~min}^{-1} 75$ vol\% $\mathrm{H}_{2}$ in $\mathrm{Ar}$ (BOC, 99.98\%). Patterns were collected for $10 \mathrm{~min}$. 


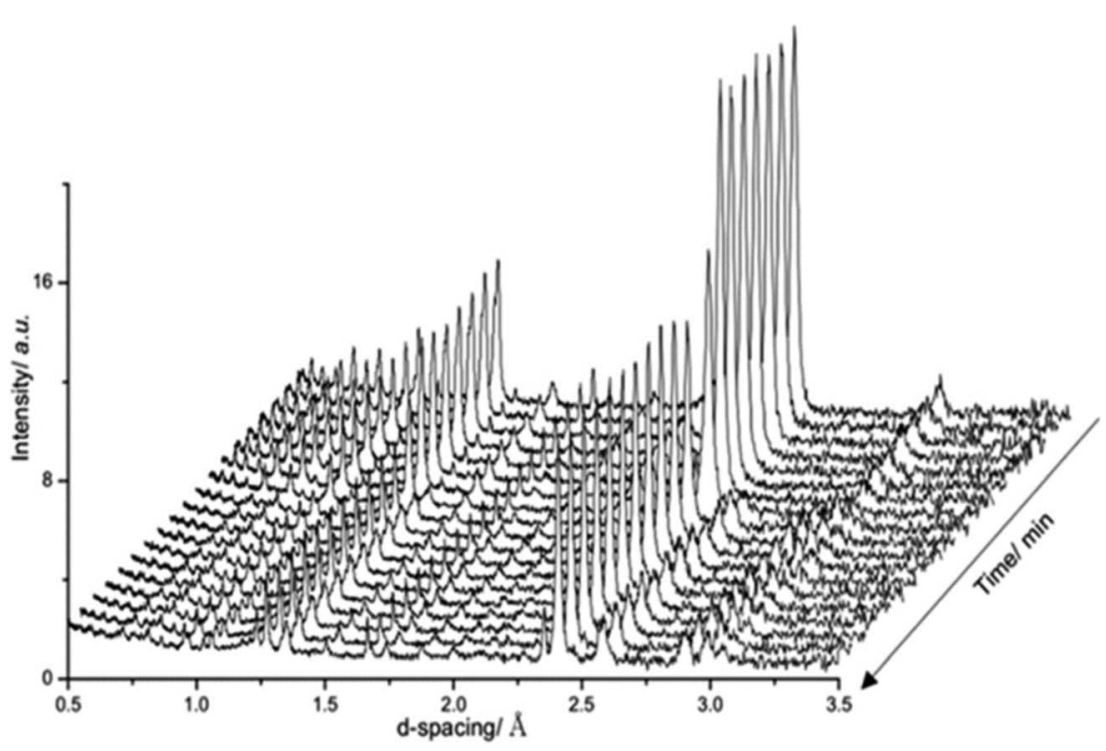

Fig. 4 Diffraction patterns of $1 \%$ wt Li/ $/ \mathrm{Mn}_{6} \mathrm{~N}_{5+x}$ during the discharge reaction at $400{ }^{\circ} \mathrm{C}$. In situ measurements were conducted under $60 \mathrm{~mL} \mathrm{~min}^{-1} 75$ vol\% $\mathrm{H}_{2}$ in $\mathrm{Ar}$ (BOC, 99.98\%). Patterns were collected for $10 \mathrm{~min}$.
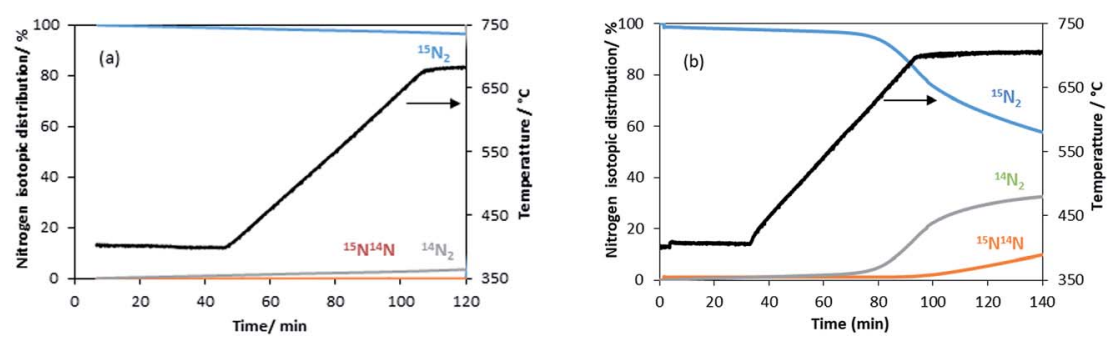

Fig. 5 Evolution of the nitrogen isotopomer distribution during the heterolytic TPNIE experiment on the manganese related materials. (a) $\theta-\mathrm{Mn}_{6} \mathrm{~N}_{5+x}$ and (b) $1 \%$ wt Li/Mn $6 \mathrm{~N}_{5+x}$.

that, under these conditions, $\theta-\mathrm{Mn}_{6} \mathrm{~N}_{5+x}$ is not able to undergo the exchange process between isotopically labelled ${ }^{15} \mathrm{~N}_{2}$ and surface/bulk nitrogen. However, when a similar temperature-programmed experiment was performed with the lithium doped system, a major difference in the exchange profile can be observed. The results are presented in Fig. 5(b), where it can be seen that there was a significant decrease in the concentration of ${ }^{15} \mathrm{~N}_{2}$ as a function of temperature, which is offset by the production of ${ }^{14} \mathrm{~N}_{2}$ and ${ }^{14} \mathrm{~N}^{15} \mathrm{~N}$. In parallel, an increase in the total pressure of the system occurred. The increase in the system pressure, beyond that normally expected in a closed loop due to increasing temperature, can be tentatively explained by decomposition producing ${ }^{14} \mathrm{~N}_{2}$ at the beginning of the process.

A similar exchange pattern was observed during the heterolytic isothermal nitrogen exchange experiment at $700{ }^{\circ} \mathrm{C}$, as presented in Fig. 6. During the exchange reaction, a significant reduction in ${ }^{15} \mathrm{~N}_{2}$ concentration was observed 


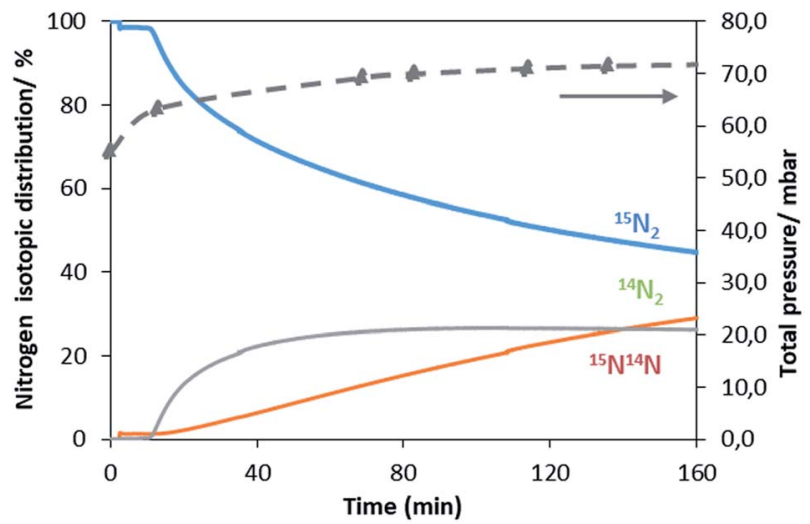

Fig. 6 Evolution of the nitrogen isotopomer distribution and of the total pressure during the heterolytic isothermal nitrogen exchange experiment at $700{ }^{\circ} \mathrm{C}$ on $1 \%$ wt Li/ $\mathrm{Mn}_{6} \mathrm{~N}_{5+x}$.

accompanied by production of ${ }^{14} \mathrm{~N}_{2}$ during the first 40 minutes of reaction whilst ${ }^{14} \mathrm{~N}^{15} \mathrm{~N}$ was only detected following ${ }^{14} \mathrm{~N}_{2}$ production. Simultaneously, an increase in the system pressure was observed during the initial stage of the experiment. This suggests possible reduction of the system prior to the activation of its exchange activity. In summary, the activation process is very limited in the nondoped system. However, a pronounced effect of lithium dopant upon the nitrogen activation process is observed, which may be a result of lowering of the nitrogen vacancy formation energy (NVFE) as suggested by the DFT calculations detailed later.

3.2.2. $\quad H_{2}$ activation process. Hydrogen activation was studied via the $H_{2} / D_{2}$ exchange reaction at $200{ }^{\circ} \mathrm{C}$ and $250{ }^{\circ} \mathrm{C}$. The results of the exchange experiments are presented in Fig. 7.

In the case of the non-doped manganese nitride sample, the $\mathrm{H}_{2} / \mathrm{D}_{2}$ exchange reaction results indicate that at $200{ }^{\circ} \mathrm{C}$ the exchange starts occurring only at a very low rate and only a small amount of HD was detected (Fig. 7(a)). Conducting the exchange at $250{ }^{\circ} \mathrm{C}$ slightly increases the exchange rate (Fig. $7($ b)). Hydrogen is usually expected to spontaneously dissociate on transition metal surfaces at low temperature $\left(<200{ }^{\circ} \mathrm{C}\right)$. However, absence of the $\mathrm{H}_{2} / \mathrm{D}_{2}$ exchange reaction on $\theta$ $\mathrm{Mn}_{6} \mathrm{~N}_{5+x}$ at room temperature and up to $150{ }^{\circ} \mathrm{C}$ was found. In the lithium promoted system, the exchange occurred at a very low rate at $200{ }^{\circ} \mathrm{C}$, in a very similar manner to the non-doped system (Fig. 7(c)). However, at $250{ }^{\circ} \mathrm{C}$ the presence of lithium accelerated the $\mathrm{H}_{2} / \mathrm{D}_{2}$ equilibration reaction compared to its non-doped counterpart, as can be seen from the significant increase in the concentration of HD produced (Fig. 7(d)). This observation indicates that at $250{ }^{\circ} \mathrm{C}$, the lithium dopant lowers the barrier for hydrogen dissociation and possibly increases the number of sites where $\mathrm{H}_{2} / \mathrm{D}_{2}$ can adsorb dissociatively via lithium-mediated chemisorption.

\subsection{DFT study of $\mathbf{H}_{2}$ and $\mathbf{N}_{2}$ adsorption on manganese nitride materials}

In order to elucidate the role of $\mathrm{Li}$ in the reactivity of manganese nitride in ammonia synthesis, $\mathrm{H}_{2}$ and $\mathrm{N}_{2}$ activation has been studied by DFT calculations. 


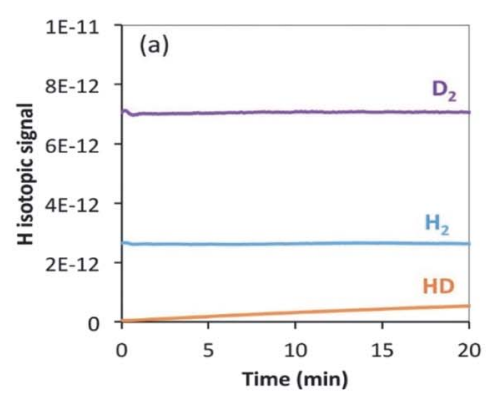

Paper
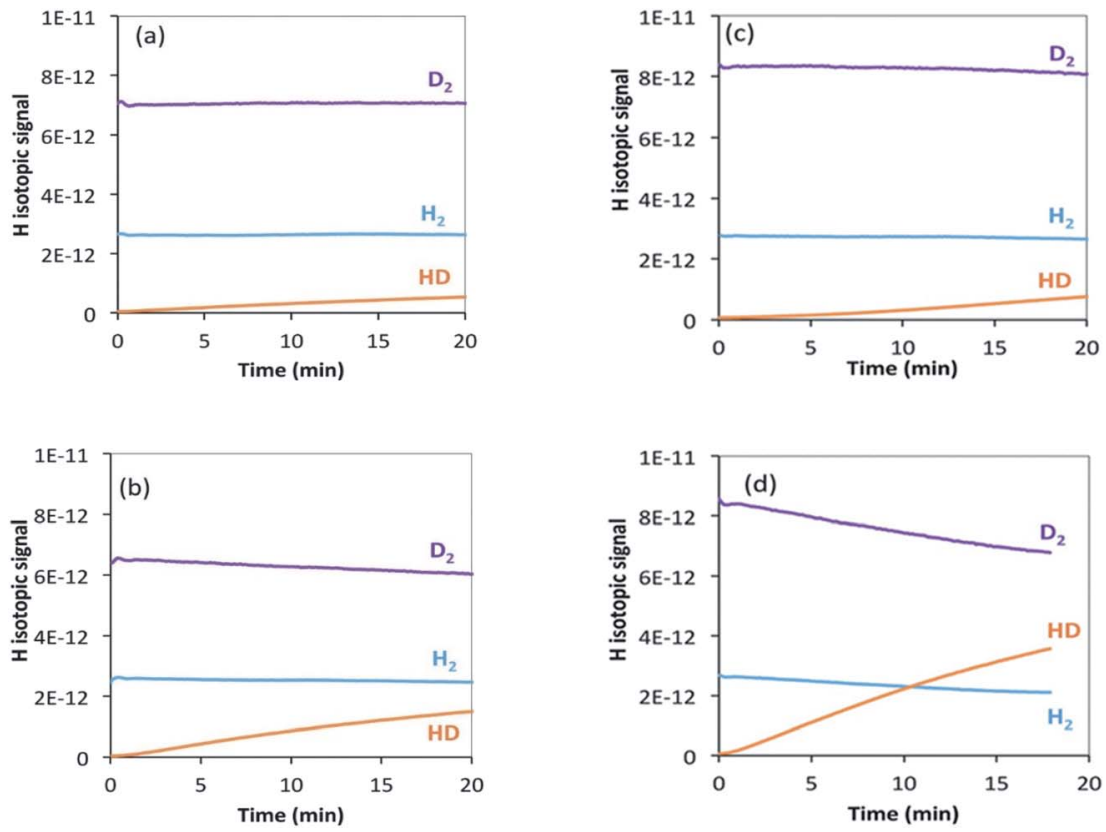

Fig. $7 \mathrm{H}_{2} / \mathrm{D}_{2}$ exchange reaction on (a) $\theta-\mathrm{Mn}_{6} \mathrm{~N}_{5+x}$ at $200{ }^{\circ} \mathrm{C}$, (b) $\theta-\mathrm{Mn}_{6} \mathrm{~N}_{5+x}$ at $250{ }^{\circ} \mathrm{C}$, (c) $1 \%$ wt Li/Mn ${ }_{6} \mathrm{~N}_{5+x}$ at $200{ }^{\circ} \mathrm{C}$, (d) $1 \%$ wt Li/Mn ${ }_{6} \mathrm{~N}_{5+x}$ at $250{ }^{\circ} \mathrm{C}$.

3.3.1. DFT study of $\mathbf{H}_{2}$ and $\mathbf{N}_{2}$ adsorption on $\mathbf{M n}_{6} \mathbf{N}_{5+x} \cdot \mathrm{H}_{2}$ and $\mathrm{N}_{2}$ adsorption and activation have already been reported in an earlier study. ${ }^{23}$ Various adsorption energies were calculated in relation to $\mathrm{N}_{2}$ adsorption on non-doped $\mathrm{Mn}_{6} \mathrm{~N}_{5+x}$. In this study, the adsorption and the activation of $\mathrm{N}_{2}$ have been investigated on the (111), (111)', (110) and (100) surfaces, ${ }^{23}$ and the adsorption energies were found to range between $-5 \mathrm{~kJ} \mathrm{~mol}^{-1}$ and $-43 \mathrm{~kJ} \mathrm{~mol}^{-1}$ in most cases. However, a value of $-120 \mathrm{~kJ} \mathrm{~mol}^{-1}$ was reported for the (111) surface. These results indicate that the adsorption of $\mathrm{N}_{2}$ is mostly molecular in the case of $\mathrm{Mn}_{6} \mathrm{~N}_{5+x}$ and only the (111) surface can effectively chemisorb nitrogen. Although the adsorption energy is very large, $\mathrm{N} \equiv \mathrm{N}$ activation was found to be less than $4 \%$ (in terms of bond length elongation). Hydrogen adsorption was found to be exclusively molecular with adsorption energies varying between -81 and $-84 \mathrm{~kJ} \mathrm{~mol}^{-1}$.

3.3.2. DFT study of $\mathbf{H}_{2}$ and $\mathbf{N}_{2}$ adsorption on $\eta-\mathbf{M n}_{3} \mathbf{N}_{2}$. The adsorption of $\mathrm{N}_{2}$ and $\mathrm{H}_{2}$ was studied on $\eta-\mathrm{Mn}_{3} \mathrm{~N}_{2}$ surfaces using periodic DFT-D3 calculations (Table 2). It is interesting to note that $\eta-\mathrm{Mn}_{3} \mathrm{~N}_{2}$ has a similar structure to $\mathrm{Mn}_{6} \mathrm{~N}_{5+x}$ with an ordered vacancy array (i.e. intrinsic $\mathrm{N}$-vacancies) which may play an important role in $\mathrm{N} \equiv \mathrm{N}$ activation. The adsorption and activation of nitrogen on $\eta-\mathrm{Mn}_{3} \mathrm{~N}_{2}$ were studied on the (100) surface as it was identified previously as the preferred surface termination. The adsorption properties were calculated at the five symmetry unique sites of the (100) surface as depicted in Scheme 1, and the values are presented in Table 2 . The adsorption energies of $\mathrm{N}_{2}$ were found to be very low, varying between $-3.6 \mathrm{~kJ} \mathrm{~mol}^{-1}$ and $-48 \mathrm{~kJ} \mathrm{~mol}^{-1}$. In fact, the adsorption energy for most adsorption sites (i.e. N1, N2, N4 and N5) was low, being between -3.6 and $-10.8 \mathrm{~kJ} \mathrm{~mol}^{-1}$ and only reaching $-48 \mathrm{~kJ} \mathrm{~mol}^{-1}$ for $\mathrm{N}_{2}$ adsorption at the 


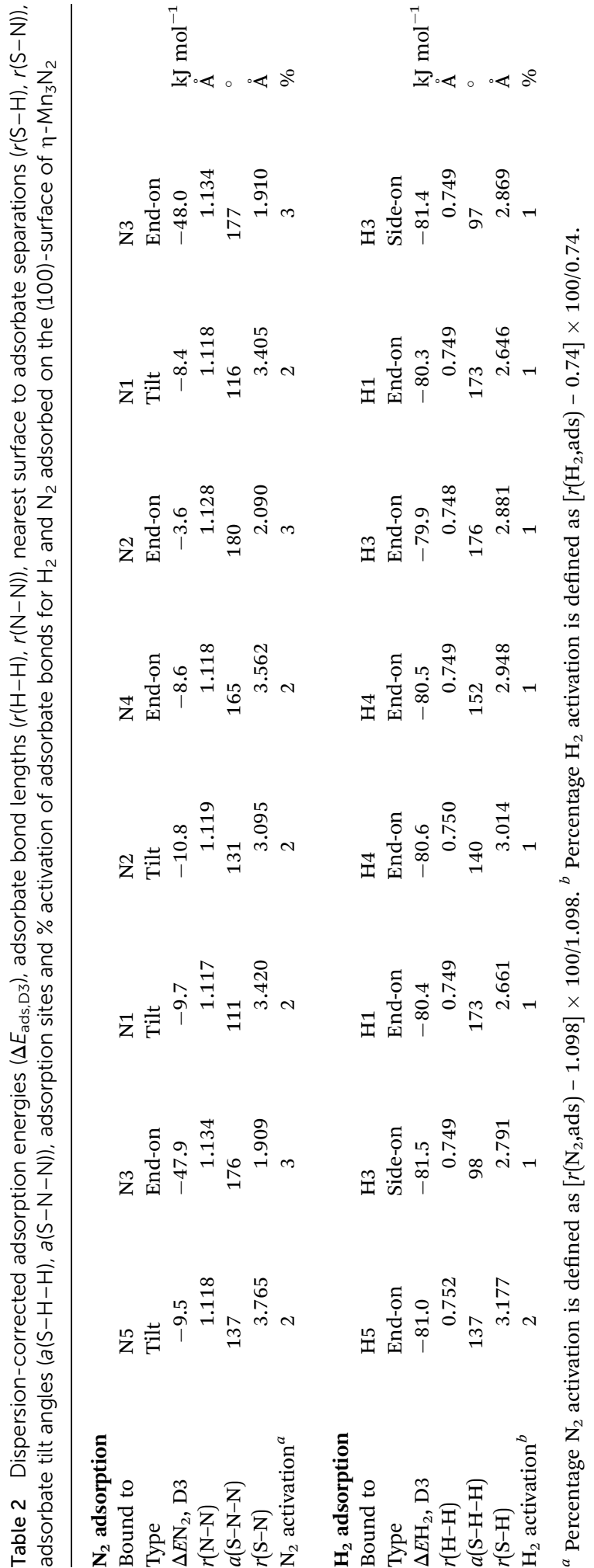


$\mathrm{Mn}(2)$ site. In contrast with the situation for $\mathrm{N}_{2}, \mathrm{H}_{2}$ was found to interact more strongly with $\eta-\mathrm{Mn}_{3} \mathrm{~N}_{2}$. The calculated adsorption energies ranged between $-79.9 \mathrm{~kJ} \mathrm{~mol}^{-1}$ and $-81.5 \mathrm{~kJ} \mathrm{~mol}^{-1}$. In this case, $\mathrm{H}_{2}$ adsorption is likely to be molecular and activated adsorption is not expected, at least for the studied (100) surface. A parallel can be established with the adsorption energy we previously reported for $\mathrm{H}_{2}$ adsorption over $\mathrm{Ta}_{3} \mathrm{~N}_{5}-(100)$, (010) and (001) surfaces, which was also reported as being mainly molecular.

In summary, hydrogen was found to adsorb end-on on N (H1) and $\mathrm{Mn}(1)$ (H2), changing to side-on for $\mathrm{Mn}(2)$ (H3). It can also adsorb end-on on a two-fold $\mathrm{Mn}$ bridge (H4) and a four-fold Mn hollow (H5). Nitrogen adsorbs in the tilt configuration on N (N1), Mn(1) (N2) and a four-fold Mn hollow (N5). We have previously attributed the three adsorption modes to the symmetry of the molecular orbitals closest to the highest occupied molecular orbital of N2. ${ }^{24}$ Nitrogen can also adsorb end-on on a Mn atom (N3) and a Mn-Mn bridge (N4).

3.3.3. Role of $\mathbf{L i}$ in $\mathbf{H}_{2}$ and $\mathbf{N}_{2}$ activation. The role of lithium dopant in the reactivity of manganese nitride was studied using $\eta-\mathrm{Mn}_{3} \mathrm{~N}_{2}$ as the host structure. The choice of $\eta-\mathrm{Mn}_{3} \mathrm{~N}_{2}$ was motivated by the results obtained from the in situ neutron diffraction studies detailed above, for which the predominant phase at $T$ $\geq 300{ }^{\circ} \mathrm{C}$ is $\eta-\mathrm{Mn}_{3} \mathrm{~N}_{2}$.

3.3.3.1 Lithium adsorption on the $\eta-M n_{3} N_{2}$ surface. The adsorption of Li on $\eta$ $\mathrm{Mn}_{3} \mathrm{~N}_{2}$ has been studied by DFT calculations (Fig. 8). Five different adsorption configurations for $\mathrm{Li}$ on $\eta-\mathrm{Mn}_{3} \mathrm{~N}_{2}$ have been identified and are depicted in Scheme 2. In particular, we find that Li chemisorbs strongly $\left(-174 \mathrm{~kJ} \mathrm{~mol}^{-1}\right.$ to $-247 \mathrm{~kJ} \mathrm{~mol}^{-1}$ ) on each of the eight symmetry unique adsorption sites of the (100) surface. The adsorption of $\mathrm{Li}$ was found to be non-selective towards $\mathrm{Mn}$ or $\mathrm{N}$. Therefore, Li may electronically affect both $\mathrm{Mn}$ and $\mathrm{N}$ when used as a dopant. This behaviour is in contrast with our observations for Co doped $\mathrm{Ta}_{3} \mathrm{~N}_{5}$, for which Co

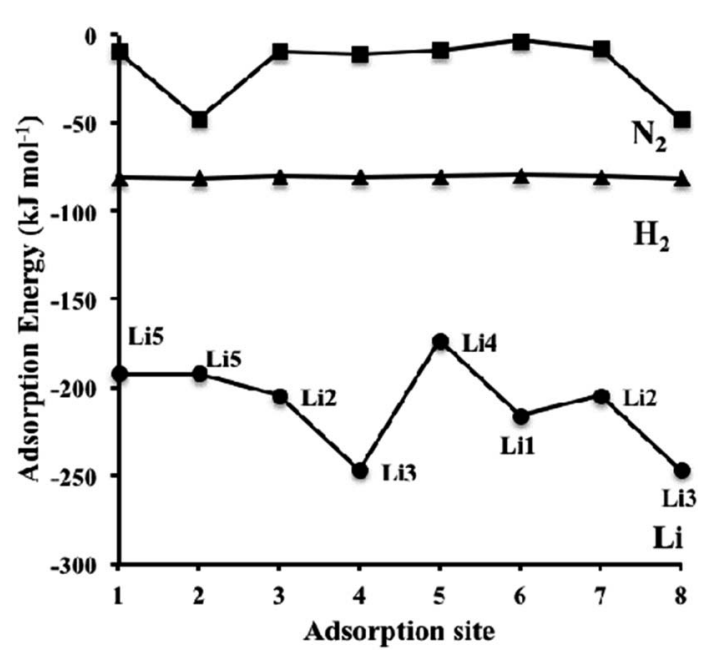

Fig. 8 Dispersion-corrected (revPBE-D3) adsorption energies of Li (circles), $\mathrm{H}_{2}$ (triangles) and $N_{2}$ (squares) over the symmetry unique adsorption sites on $\eta-M n_{3} N_{2}$ depicted in Scheme 1 (for $\mathrm{H}_{2}$ and $\mathrm{N}_{2}$ ). The data for Li corresponds to the adsorption site configuration depicted in Scheme 2. Lines connecting the data points have been drawn to guide the eye. 


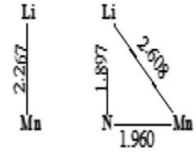

Lil
Li2

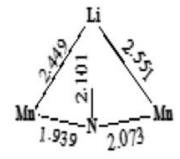

Li3

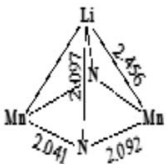

Li4

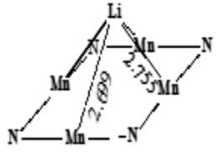

LiB

Scheme 2 Various binding configurations of Li atoms on $\eta-M n_{3} N_{2}$ along with selected bond lengths given in $\AA$.

was found to adsorb mostly on N-rich sites, which may be attributed to the metallic property of $\mathrm{Co}^{23}$

The adsorption energy of $\mathrm{Li}$ is much stronger than that of $\mathrm{H}_{2}$ or $\mathrm{N}_{2}$, as shown in Fig. 8; therefore, the adsorption of the reactants for ammonia synthesis is predicted not to perturb significantly the structure of the Li- $\eta-\mathrm{Mn}_{3} \mathrm{~N}_{2}$ phase at low temperatures. Furthermore, it was observed that $\mathrm{Li}$ decreases the number of unpaired electrons on the $\mathrm{Mn}$ atoms as indicated by changes in their effective magnetic moment. In particular, we observe a decrease by an average value of 0.9 $\mu_{\mathrm{B}}$, indicating pairing of the lone electron of Li with the unoccupied d-orbitals of the $\mathrm{Mn}$ atoms. This observation suggests that the promoting effect of lithium on $\eta-\mathrm{Mn}_{3} \mathrm{~N}_{2}$ is electronic in nature, which is a result of electron injection from Li into the unoccupied d-states of $\mathrm{Mn}$.

3.3.3.2 Nitrogen vacancy formation energy in Li doped $\eta-M n_{3} N_{2}$. The evolution of the nitrogen vacancy formation energy (NVFE) was studied as a function of coverage of $\mathrm{Li}$ on the surface vacancies of $\eta-\mathrm{Mn}_{3} \mathrm{~N}_{2}$. The results are presented in Fig. 9.

In the absence of $\mathrm{Li}$, the NVFE of $\eta-\mathrm{Mn}_{3} \mathrm{~N}_{2}$ in bulk is $1.3 \mathrm{eV}$, which is identical to the NVFE at the (100) surface. This indicates some degree of rehybridization of the bonding orbitals of surface-N compared to bulk-N in order to keep the NVFE of the 6 -fold bound $\mathrm{N}$ in the bulk the same as the 5 -fold bound $\mathrm{N}$ on the surface. $\mathrm{A}$ comparison of the band structures of bulk and surface $\eta-\mathrm{Mn}_{3} \mathrm{~N}_{2}$ shows an up-shift of the $\mathrm{N} 2 \mathrm{~s}$ band by about $0.5 \mathrm{eV}$ in the slab DOS. Having examined the NVFE at
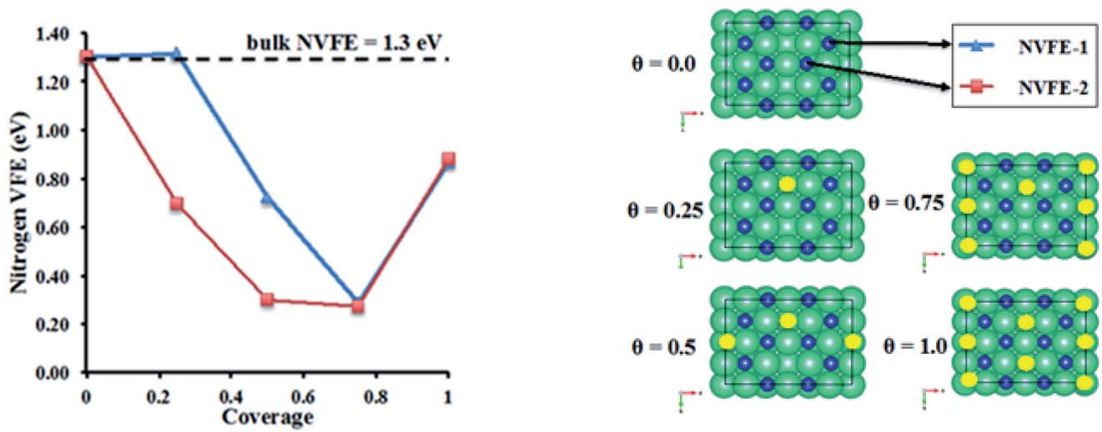

Fig. 9 Nitrogen vacancy formation energy (NVFE) as a function of coverage of $\mathrm{Li}$ promoters on $\eta-M_{3} N_{2}$. Li: yellow, Mn: green and $\mathrm{N}$ : blue. The NVFE was calculated based on the following equation: NVFE $=E_{\mathrm{Nvac}}+1 / 2 E_{\mathrm{N}_{2}}-E_{\text {slab }}$ where $E_{\mathrm{Nvac}}$ is the total energy of the surface that has the nitrogen vacancy, $E_{\mathrm{N}_{2}}$ is the total energy of $\mathrm{N}_{2}$, and $E_{\text {slab }}$ is the total energy of the defect-free slab. 
two sites (i.e. NVFE-1 and NVFE-2), it was found that the location of the Li promoter with respect to the prospective $\mathrm{N}$-vacancy determines the reducing effect on the NVFE (i.e. promoting effect). In particular, it appears that there is a promotional effect only when the Li promoter is separated from $\mathrm{N}$, and a vacancy is formed by a $\mathrm{Mn}-\mathrm{Mn}$ bond rather than a $\mathrm{Mn}$ atom (see $\theta=0.25$ in Fig. 9 for the position of Li compared to $\mathrm{N}$ for NVFE-1 and NVFE-2). Intriguingly, both NVFE curves (i.e. NVFE-1 and NVFE-2) as a function of coverage show that there is an optimum surface concentration for the Li atoms so that the promoting effect is maximized, which corresponds to a coverage of $\theta=0.75$ and a ratio of $\mathrm{Mn}: \mathrm{Li}$ surface atoms (i.e. $\mathrm{Mn}_{\text {surf }}: \mathrm{Li}_{\text {surf }}$ ) of $4: 1$. Higher Li coverage appears to increase the NVFE and would additionally prevent the reactants from interacting directly with the $\eta-\mathrm{Mn}_{3} \mathrm{~N}_{2}$ phase. Low Li coverage results in a NVFE which is almost $1 \mathrm{eV}$ higher than that at $\theta=0.75$. The NVFE is an indication of the energy required to remove surface $\mathrm{N}$ via reactions with hydrogen:

$$
\text { Li- } \eta-\mathrm{Mn}_{3} \mathrm{~N}_{2}+\frac{3}{2} \mathrm{H}_{2} \leftrightarrow \text { Li- } \eta-\mathrm{Mn}_{3} \mathrm{~N}_{2-x}+x \mathrm{NH}_{3}
$$

On this basis, we expect that a Li surface concentration of $\mathrm{Mn}_{\text {surf }}: \mathrm{Li}_{\text {surf }}=4: 1$ to be specific for promoting ammonia synthesis reactions on $\eta-\mathrm{Mn}_{3} \mathrm{~N}_{2}$.

\subsection{Integration of experimental work and computational modelling in $\mathrm{Li}$ doped manganese nitride materials}

The ability of manganese to activate nitrogen to form an array of nitrides with different structure and nitrogen content has attracted much interest for the development of two-stage reactants for ammonia synthesis by chemical looping. However, unless they are doped with $\mathrm{Li}$, the reactivity of manganese nitrides was found to be limited in the low temperature range. The stability of manganese nitride was clearly observed by in situ neutron diffraction studies (Fig. 3) where no changes in the structural properties of $\theta-\mathrm{Mn}_{6} \mathrm{~N}_{5+x}$ or of $\eta-\mathrm{Mn}_{3} \mathrm{~N}_{2}$ (present as a minor phase) were observed during the discharge reaction conducted using 60 $\mathrm{mL} \min ^{-1} 75 \mathrm{vol} \% \mathrm{H}_{2}$ in $\mathrm{Ar}$ (BOC, 99.98\%). To extend the study, $\mathrm{H}_{2}$ and $\mathrm{N}_{2}$ adsorption and activation have been studied using isotopic exchange and DFT modelling. The adsorption energies as evaluated using periodic DFT-D3 calculations showed that $\mathrm{N}_{2}$ only weakly interacted with $\eta-\mathrm{Mn}_{3} \mathrm{~N}_{2}$ and $\theta-\mathrm{Mn}_{6} \mathrm{~N}_{5+x}$ (with adsorption energy ranging between $-3.6 \mathrm{~kJ} \mathrm{~mol}^{-1}$ and $-48 \mathrm{~kJ} \mathrm{~mol}^{-1}$ ), ruling out an activated adsorption process. These results agree with those of nitrogen isotopic exchange where no significant $\mathrm{N}_{2}$ activation and dissociation was observable under the reaction conditions applied. The energy relating to $\mathrm{H}_{2}$ adsorption was also calculated for the non-doped nitrides and ranged between $-79.9 \mathrm{~kJ} \mathrm{~mol}^{-1}$ and $-81.5 \mathrm{~kJ} \mathrm{~mol}^{-1}$. Despite the stronger interaction, $\mathrm{H}_{2}$ was found to be adsorbed mainly in a molecular from. The $\mathrm{H}_{2}$ isotopic exchange reaction also showed limited activation of $\mathrm{H}_{2} / \mathrm{D}_{2}$ at temperatures below $250{ }^{\circ} \mathrm{C}$ and only limited production of HD was observed, indicating an important energy barrier for the dissociative chemisorption of $\mathrm{H}_{2}$ on manganese nitride materials.

Li doping was found to be critical in the reactivity of manganese nitride during the discharge reaction. Doping with a low level of Li was found to enhance greatly the production of ammonia during the discharge reaction. The same positive effect was observed in the $\mathrm{N}_{2}$ and $\mathrm{H}_{2}$ isotopic exchange reactions. In situ neutron 
diffraction studies have shown that the reaction of $1 \%$ wt $\mathrm{Li} / \mathrm{Mn}_{6} \mathrm{~N}_{5+x}$ is accompanied by significant structural changes. The reduction process started with nitrogen vacancy creation at low temperature followed by the transformation of $\theta$ $\mathrm{Mn}_{6} \mathrm{~N}_{5+x}$ into $\eta-\mathrm{Mn}_{3} \mathrm{~N}_{2}$ at $\sim 300{ }^{\circ} \mathrm{C}$. A parallel might be established with the heterolytic TPNIE experimental results, where an increase in ${ }^{14} \mathrm{~N}_{2}$ concentration and total pressure occurred prior to the initiation of the ${ }^{15} \mathrm{~N}_{2} /{ }^{14} \mathrm{~N}_{2}$ exchange reaction. Taking into account that the differences between the $\theta-\mathrm{Mn}_{6} \mathrm{~N}_{5+x}$ and $\eta$ $\mathrm{Mn}_{3} \mathrm{~N}_{2}$ structures lie in the presence of an ordered nitrogen vacancy array, the nitrogen vacancy formation energy as a function of $\mathrm{Li}$ concentration has been calculated (Fig. 9). The presence of Li was found to decrease the NVFE from $1.3 \mathrm{eV}$ (in the absence of dopant, i.e. $\theta=0)$ to $0.3 \mathrm{eV}(\theta=0.75)$. Hence, doping with $\mathrm{Li}$ enhances greatly the formation of nitrogen vacancies, lowering the energy required to remove $\mathrm{N}$ from the surface. Such nitrogen vacancy sites have been reported as active sites for $\mathrm{N}_{2}$ activation in the case of $\mathrm{Co}_{3} \mathrm{Mo}_{3} \mathrm{~N},{ }^{24}$ and this could explain the reactivity of doped manganese nitrides.

It is interesting to draw comparisons between the present study and previous investigations relating to manganese nitride being applied for solar ammonia synthesis upon reaction with water ${ }^{25}$ and the thermochemical synthesis of ammonia and syngas from natural gas at atmospheric pressure. ${ }^{6}$ In the former investigation, the inclusion of transition metal dopants to modify the reactivity of lattice nitrogen to generate desirable processes and redox properties was modelled using electronic structure theory, and experimental validation was undertaken using iron doped manganese nitride. ${ }^{25}$

\section{Conclusion}

A combined experimental and computational modelling based study has been undertaken to provide additional understanding of the beneficial role of lithium doping in the enhancement of ammonia synthesis upon hydrogenation of manganese nitride. Powder neutron diffraction demonstrates that lithium doping enhances the phase fraction of $\theta-\mathrm{Mn}_{6} \mathrm{~N}_{5+x}$ which was shown to progressively lose nitrogen as a function of temperature upon reduction, yielding a structure related to $\eta-\mathrm{Mn}_{3} \mathrm{~N}_{2}$. The observation of enhanced lattice nitrogen reactivity upon lithium doping was apparent in isotopic exchange studies, which also demonstrated that lithium enhanced hydrogen activation. Consistent with these experimental observations, in computational modelling studies, lithium doping is found to reduce the nitrogen vacancy formation energy which is anticipated to lead to enhanced reactivity.

\section{Conflicts of interest}

There are no conflicts to declare.

\section{Acknowledgements}

The authors acknowledge the support from the PRESTIGE H2020 program and the financial support from MOPGA and the Region Haut de France Research Funding Program. This study was supported by EPSRC funding (EP/L026317/1, EP/L02537X/ 1). Via our membership of the UK's HEC Materials Chemistry Consortium, which is funded by EPSRC (EP/L000202/1), this work used the ARCHER UK National 
Supercomputing Service (http://www.archer.ac.uk). The authors acknowledge the use of the UCL Legion and Grace High Performance Computing Facility (Legion@UCL, Grace@UCL), and associated support services, in the completion of this work. European Union (ERDF) and Région Nouvelle Aquitaine region are also gratefully acknowledged for their financial support.

\section{Notes and references}

1 S. Laassiri, C. D. Zeinalipour-Yazdi, C. R. A. Catlow and J. S. J. Hargreaves, Catal. Today, 2017, 286, 147-155.

2 G. E. Veitch, K. L. Bridgwood and S. V. Ley, Org. Lett., 2008, 10, 3623-3625.

3 D. McKay, D. H. Gregory, J. S. J. Hargreaves, S. M. Hunter and X. Sun, Chem. Commun., 2007, 3051-3053.

4 I. AlShibane, A. Daisley, J. S. J. Hargreaves, A. L. Hector, S. Laassiri, J. L. Rico and R. I. Smith, ACS Sustainable Chem. Eng., 2017, 5, 9214-9222.

5 S. Laassiri, C. D. Zeinalipour-Yazdi, C. R. A. Catlow and J. S. Hargreaves, Appl. Catal., B, 2018, 223, 60-66.

6 M. G. Heidlage, E. A. Kezar, K. C. Snow and P. H. Pfromm, Ind. Eng. Chem. Res., 2017, 56, 14014-14024.

7 M. Widenmeyer, T. C. Hansen, A. Leineweber, A. Weidenkaff and R. Niewa, $Z$. Anorg. Allg. Chem., 2017, 643, 1929-1938.

8 A. Leineweber, R. Niewa, H. Jacobs and W. Kockelmann, J. Mater. Chem., 2000, 10, 2827-2834.

9 R. Michalsky, A. M. Avram, B. A. Peterson, P. H. Pfromm and A. A. Peterson, Chem. Sci., 2015, 6, 3965-3974.

10 P. Wang, F. Chang, W. Gao, J. Guo, G. Wu, T. He and P. Chen, Nat. Chem., 2017, 9, 64.

11 R. Schlögl, Angew. Chem., Int. Ed., 2003, 42, 2004-2008.

12 A. Miura, T. Takei and N. Kumada, Inorg. Chem., 2013, 52, 11787-11791.

13 S. Laassiri, N. Bion, D. Duprez, S. Royer and H. Alamdari, Phys. Chem. Chem. Phys., 2014, 16, 4050-4060.

14 B. Toby, J. Appl. Crystallogr., 2001, 34, 210-213.

15 A. C. Larson and R. B. V. Dreele, Gsas General Structure Analysis System, Los Alamos National Laboratory, 2000, pp. 86-748.

16 H. J. Monkhorst and J. D. Pack, Phys. Rev. B: Solid State, 1976, 13, 5188-5192.

17 G. Kresse and J. Hafner, Phys. Rev. B: Condens. Matter Mater. Phys., 1993, 47, 558-561.

18 G. Kresse and J. Furthmüller, Phys. Rev. B: Condens. Matter Mater. Phys., 1996, 54, 11169-11186.

19 J. P. Perdew, K. Burke and M. Ernzerhof, Phys. Rev. Lett., 1996, 77, 3865-3868.

20 G. Kresse and D. Joubert, Phys. Rev. B: Condens. Matter Mater. Phys., 1999, 59, 1758-1775.

21 P. E. Blöchl, Phys. Rev. B: Condens. Matter Mater. Phys., 1994, 50, 17953-17979.

22 S. Grimme, J. Antony, S. Ehrlich and H. Krieg, J. Chem. Phys., 2010, 132, 154104.

23 C. D. Zeinalipour-Yazdi, Phys. Chem. Chem. Phys., 2019, 21, 19365-19377.

24 C. D. Zeinalipour-Yazdi, J. S. J. Hargreaves and C. R. A. Catlow, J. Phys. Chem. C, 2015, 119, 28368-28376.

25 R. Michalsky, P. H. Pfromm and A. Steinfeld, Interface Focus, 2015, 5, 20140084. 